\title{
Balanced Labeling and Balance Index Set of One Point Union of Two Complete Graphs
}

\author{
Pradeep G Bhat \\ Department of Mathematics \\ Manipal Institute of Technology \\ Manipal, Karnataka, India
}

\author{
Devadas Nayak C \\ Department of Mathematics \\ Manipal Institute of Technology \\ Manipal, Karnataka, India
}

\begin{abstract}
Let $G$ be a graph with vertex set $V(G)$ and edge set $E(G)$, and consider the set $A=\{0,1\}$. A labeling $f: V(G) \rightarrow A$ induces a partial edge labeling $f^{*}: E(G) \rightarrow A$ defined by $f^{*}(x y)=f(x)$, if and only if $f(x)=f(y)$, for each edge $x y \in E(G)$. For $i \in A$, let $v_{f}(i)=|\{v \in V(G): f(v)=i\}|$ and $e_{f^{*}}(i)=\left|e \in E(G): f^{*}(e)=i\right|$. A labeling $f$ of a graph $G$ is said to be friendly if $\left|v_{f}(0)-v_{f}(1)\right| \leq 1$. A friendly labeling is called balanced if $\left|e_{f^{*}}(0)-e_{f^{*}}(1)\right| \leq 1$. The balance index set of the graph $G, \operatorname{Bl}(G)$, is defined as $\left\{\left|e_{f^{*}}(0)-e_{f^{*}}(1)\right|\right.$ : the vertex labeling $f$ is friendly $\}$. We provide balanced labeling and balance index set of one point union of two complete graphs.
\end{abstract}

\section{General Terms:}

Balance index set of graph $\mathrm{G}$ is denoted by $\mathrm{BI}(\mathrm{G})$ and one point union of two complete graphs is denoted by $K_{m} \cdot K_{n}$.

\section{Keywords:}

Vertex labeling, Friendly labeling, Cordial labeling, Balanced labeling and Balance index set.

\section{INTRODUCTION}

We begin with simple, finite, connected and undirected graph $G=(V, E)$. Here elements of set $V$ and $E$ are known as vertices and edges respectively. If $G$ and $H$ are graphs with the property that the identification of any vertex of graph $\mathrm{G}$ with an arbitrary vertex of graph $\mathrm{H}$ results in a unique graph(up to isomorphism), then we write $G \cdot H$ for this graph. This graph is known as one point union of two graphs. For all other terminologies and notations we follow Harary [?] .

If the vertices of the graph are assigned values subject to certain conditions is known as graph labeling.

Most interesting graph labeling problems have three important characteristics.

* a set of numbers from which the labels are chosen;

$*$ a rule that assigns a value to each edge;

$*$ a condition that these values must satisfy.

For detail survey on graph labeling one can refer Galian [?]. Vast amount of literature is available on different types of graph labeling. According to Beineke and Hegde [?] graph labeling serves as a frontier between number theory and structure of graphs.

Labeled graphs are becoming an increasingly useful family of mathematical models for a broad range of applications.
The qualitative labelings of graph elements have inspired research in diverse fields of human enquiry such as conflict resolution in social psychology, electrical circuit theory and energy crisis. Quantitative labelings of graphs have led to quite intricate fields of applications such as coding theory problems, including the design of good radar location codes, synch-set codes, missile guidance codes and convolution codes with optimal auto-correlation properties. Labeled graphs have also been applied in determining ambiguities in X-Ray crystallographic analysis, to design communication network addressing systems, to determine optimal circuit layouts and radio-astronomy, etc.

In 1986 Cahit [?] introduced cordial graph labeling. A function $f$ from $V(G)$ to $\{0,1\}$, where for each edge $x y, f^{*}(x y)=|f(x)-f(y)|, v_{f}(i)$ is the number of vertices $v$ with $f(v)=i$, and $e_{f^{*}}(i)$ is the number of edges $e$ with $f^{*}(e)=i$ is called friendly if $\left|v_{f}(0)-v_{f}(1)\right| \leq 1$. A friendly labeling $f$ is called cordial if $\left|e_{f^{*}}(0)-e_{f^{*}}(1)\right| \leq 1$.

Lee, Liu and Tan [?] considered a new labeling problem of graph theory. A vertex labeling of $G$ is a mapping $f$ from $V(G)$ into the set $\{0,1\}$. For each vertex labeling $f$ of $G$, a partial edge labeling $f^{*}$ of $G$ is defined in the following way. For each edge $u v$ in $G$,

$$
f^{*}(u v)=\left\{\begin{array}{l}
0, \text { if } f(u)=f(v)=0 \\
1, \text { if } f(u)=f(v)=1
\end{array}\right.
$$

Note that if $f(u) \neq f(v)$, then the edge $u v$ is not labeled by $f^{*}$. Thus $f^{*}$ is a partial function from $E(G)$ into the set $\{0,1\}$. Let $\quad v_{f}(0)$ and $v_{f}(1)$ denote the number of vertices of $\mathrm{G}$ that are labeled by 0 and 1 under the mapping $f$ respectively. Likewise, let $e_{f^{*}}(0)$ and $e_{f^{*}}(1)$ denote the number of edges of $G$ that are labeled by 0 and 1 under the induced partial function $f^{*}$ respectively.

DEFINITION 1. A graph $G$ is said to be a balanced graph or balanced if there is a vertex labeling $f$ of $G$ such that $\left|v_{f}(0)-v_{f}(1)\right| \leq 1$ and $\left|v_{f^{*}}(0)-v_{f^{*}}(1)\right| \leq 1$. A graph $G$ is said to be strongly vertex-balanced if $G$ is a balanced graph and $v_{f}(0)=v_{f}(1)$. Similarly a graph $G$ is strongly edge-balanced if it is a balanced graph and $e_{f^{*}}(0)=e_{f^{*}}(1)$. If $G$ is a strongly vertex-balanced and strongly edge-balanced graph, then $G$ is a strongly balanced graph.

EXAMPLE 1. Figure 1 shows a graph with two distinct balanced labelings of graph $G$.

The following graphs are studied in [?]

(1) The path $P_{n}$ is balanced; it is strongly balanced if $n$ is even.

(2) The cycle $C_{n}$ is balanced; it is strongly balanced if $n$ is even. 

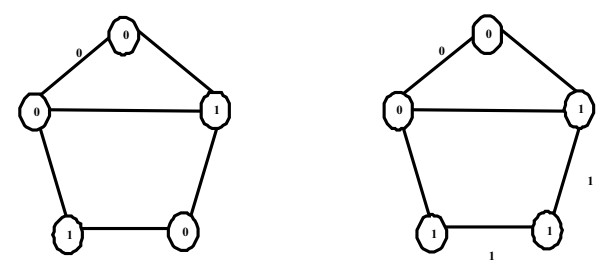

Fig. 1. Two distinct balanced labelings of graph $G$

(3) The complete graph $K_{n}$ is strongly balanced if and only if $n$ is even.

(4) The complete bipartite graph $K_{m, n}$ is balanced if and only if one of the following conditions holds,

(a) $m, n$ are even,

(b) $m, n$ are odd and $|m-n| \leq 2$

(c) one of $m$ and $n$, say $m$, is odd, $n=2 t$ and $t=-1,0$ or $1[\bmod (|m-n|)]$.

(5) If $G$ is $k$-regular with $p$ vertices, then

(a) $G$ is balanced if $p$ is odd and $k=2$;

(b) $G$ is strongly balanced if $p$ is even.

In [?] Kim, Lee, and $\mathrm{Ng}$ define the balance index set of a graph $G$ as $\operatorname{BI}(G)=\left\{\left|e_{f^{*}}(0)-e_{f^{*}}(1)\right|: f^{*}\right.$ runs over all friendly labelings $f$ of $G$ \}. The balance index set of star, double star, complete graphs, complete bipartite graphs and windmill graphs given in [?].

EXAMPLE 2. $B I(G)=\{0,1\}$.

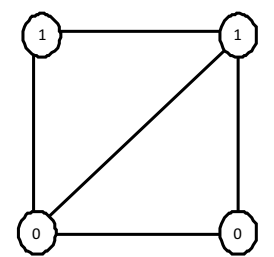

$\left|e_{f^{*}}(\mathbf{0})-e_{f^{*}}(\mathbf{1})\right|=\mathbf{0}$

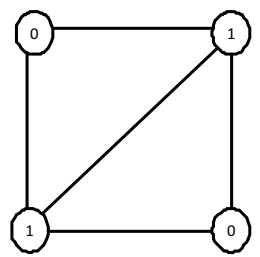

$\left|e_{f^{*}}(0)-e_{f^{*}}(1)\right|=1$
Fig. 2. Distinct friendly labelings of $G$

In number theory and combinatorics, a partition of a positive integer $n$, also called an integer partition, is a way of writing $n$ as a sum of positive integers. Two sums that differ only in the order of their summands are considered to be the same partition; if order matters then the sum becomes a composition. For example, 4 can be partitioned in five distinct ways:

$4+0$,

$3+1$,

$2+2$,

$2+1+1$,

$1+1+1+1$.

\section{BALANCED LABELING OF ONE POINT UNION OF TWO COMPLETE GRAPHS}

First we discuss Balanced Labeling of $K_{m} \cdot K_{n}$. In this paper we are using the idea of integer partition of numbers. Let $G$ be any graph with $p$ vertices. Partition of $p$ in to $\left(p_{1}, p_{2}\right)$, where $p_{1}$ and $p_{2}$ are the number of vertices labeled by ' 0 ' and ' 1 ' respectively.

THEOREM 2. The one point union of two graphs $G$ and $H$, say $G \cdot H$ with $m$ and $n$ vertices respectively, satisfies friendly labeling if and only if

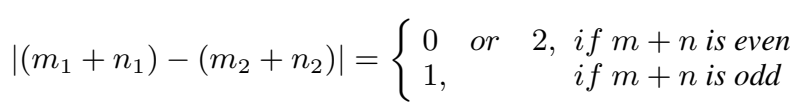

where $m=\left(m_{1}, m_{2}\right)$ and $n=\left(n_{1}, n_{2}\right)$.

Proof. Consider $G \cdot H$ with $m$ and $n$ vertices in $G$ and $H$ respectively.

Case 1. If $m+n$ is even, then $|V(G \cdot H)|=m+n-1$ which is odd. Therefore every friendly labeling $f$ satisfies the condition $\left|v_{f}(0)-v_{f}(1)\right|=1$. This gives, either $v_{f}(0)=m_{1}+n_{1}-1$ and $v_{f}(1)=m_{2}+n_{2}$ or $v_{f}(0)=m_{1}+n_{1}$ and $v_{f}(1)=m_{2}+n_{2}-1$. $\left|v_{f}(0)-v_{f}(1)\right|=1$

$\Leftrightarrow\left|\left(m_{1}+n_{1}\right)-\left(m_{2}+n_{2}\right) \pm 1\right|=1$

$\Leftrightarrow\left|\left(m_{1}+n_{1}\right)-\left(m_{2}+n_{2}\right)\right|=0$ or 2

Case 2. If $m+n$ is odd, then $|V(G \cdot H)|=m+n-1$ which is even. Therefore every friendly labeling $f$ satisfies the condition $\left|v_{f}(0)-v_{f}(1)\right|=0$. This gives, either $v_{f}(0)=m_{1}+n_{1}-1$ and $v_{f}(1)=m_{2}+n_{2}$ or $v_{f}(0)=m_{1}+n_{1}$ and $v_{f}(1)=m_{2}+n_{2}-1$. $\left|v_{f}(0)-v_{f}(1)\right|=0$

$\Leftrightarrow\left|\left(m_{1}+n_{1}\right)-\left(m_{2}+n_{2}\right) \pm 1\right|=0$

$\Leftrightarrow\left|\left(m_{1}+n_{1}\right)-\left(m_{2}+n_{2}\right)\right|=1 \quad \square$

THEOREM 3. A graph $K_{n} \cdot K_{n+1}$ is strongly balanced.

Proof. Since $|V(G)|=2 n$, every friendly labeling of $K_{n} \cdot K_{n+1}$ gives $v_{f}(0)=v_{f}(1)=n$. We label the vertices of $K_{n}$ by 0 and the remaining vertices by 1 .

Then $e_{f^{*}}(0)=e_{f^{*}}(1)=\left(\begin{array}{c}n \\ 2\end{array}\right)$. Therefore $\left|v_{f}(0)-v_{f}(1)\right|=0$ and $\left|e_{f^{*}}(0)-e_{f^{*}}(1)\right|=0$. Hence the graph $K_{n} \cdot K_{n+1}$ is strongly balanced.

THEOREM 4. A graph $K_{m} \cdot K_{n}$ is balanced, when $|m-n| \neq 1$, if

(1) Both $m$ and $n$ are even.

(2) Both $m$ and $n$ are odd with

(a) $\min \{m, n\}=3$ and $|m-n| \leq 6$

(b) $\min \{m, n\} \geq 5$ and $|m-n| \leq 2$.

(3) $m+n$ is odd and $\min \{m, n\}=$ odd with

(a) $\min \{m, n\}=3$ and for all $n$

(b) $\min \{m, n\} \geq 5$ and if there exist an integer $z=2 i(n-m)$, where $i=\left[\frac{m-1}{6}\right]$, satisfying the condition $m-3 \leq z \leq m+1$.

(4) $m+n$ is odd, $\min \{m, n\}=$ even and if there exist an integer $z=2 i(n-m)$, where $i=1,2,3, \ldots, \frac{\min \{m, n\}}{2}$ satisfying the condition $n-3 \leq z \leq n+1$.

Proof. Here $\left|V\left(K_{m} \cdot K_{n}\right)\right|=m+n-1$ and $\left|E\left(K_{m} \cdot K_{n}\right)\right|$ $=\left(\begin{array}{c}m \\ 2\end{array}\right)+\left(\begin{array}{c}n \\ 2\end{array}\right)$.

Without loss of generality we consider $m \leq n$.

(1) Since both $m$ and $n$ are even, $\left|V\left(K_{m} \cdot K_{n}\right)\right|$ is odd. Therefore every friendly labeling satisfying the condition $\left|v_{f}(0)-v_{f}(1)\right|=1$.

Consider two complete blocks $K_{m}$ and $K_{n}$ of $K_{m} \cdot K_{n}$. We label $\frac{m}{2}$ number of vertices of $K_{m}$ and $\frac{n}{2}$ number of vertices of $K_{n}$ by 1 , and the remaining vertices by 0 . Then $\left|v_{f}(0)-v_{f}(1)\right|=1$ and $\left|e_{f^{*}}(0)-e_{f^{*}}(1)\right|=0$. Hence graph $K_{m} \cdot K_{n}$ is strongly edge-balanced if both $\mathrm{m}$ and $\mathrm{n}$ are even.

(2) Since both $m$ and $n$ are odd, $\left|V\left(K_{m} \cdot K_{n}\right)\right|$ is odd. Therefore every friendly labeling satisfying the condition $\left|v_{f}(0)-v_{f}(1)\right|=1$.

(a) If $m=3$.

Since $n$ is odd, $n=2 k+1, k \geq 1$.

Then the graph $K_{3} \cdot \bar{K}_{n}$ is balanced if $\left|\left(\begin{array}{c}k+1 \\ 2\end{array}\right)-\left(\begin{array}{l}k \\ 2\end{array}\right)\right| \leq 4$ 
(b) If $m \geq 5$, then $K_{m} \cdot K_{n}$ is balanced if

$$
\begin{aligned}
& \mid\left[\left(\begin{array}{c}
\frac{m+1}{2} \\
2
\end{array}\right)+\left(\frac{n-1}{2}\right)\right] \\
& -\left[\left(\begin{array}{c}
\frac{m+1}{2} \\
2
\end{array}\right)+\left(\begin{array}{c}
\frac{n-1}{2} \\
2
\end{array}\right)\right] \mid \leq 1 \\
& \quad \Rightarrow|m-n| \leq 2 .
\end{aligned}
$$

(3) Since $m+n$ is odd, $\left|V\left(K_{m} \cdot K_{n}\right)\right|$ is even. Therefore every friendly labeling satisfying the condition $\left|v_{f}(0)-v_{f}(1)\right|=$ 0 .

(a) If $m=3$. We label two vertices of $K_{3}$ by 0 and one vertex by 1 , and half the number of vertices of $K_{n}$ by 0 and remaining half by 1 . Then $\left|e_{f^{*}}(0)-e_{f^{*}}(1)\right|=1$.

(b) If $m \geq 5$. Consider the partition of $m$ and $n$ as $\left(\frac{m-(2 i+1)}{2}, \frac{m+(2 i+1)}{2}\right)$ and $\left(\frac{n}{2}+i, \frac{n}{2}-i\right)$ respectively, where $i=\left[\frac{m-1}{6}\right]$. Then $K_{m} \cdot K_{n}$ is balanced if

$$
\begin{aligned}
& \mid\left[\left(\frac{m-(2 i+1)}{2}\right)+\left(\begin{array}{c}
\frac{n}{2}+i \\
2
\end{array}\right)\right] \\
& \quad-\left[\left(\frac{m+(2 i+1)}{2}\right)+\left(\begin{array}{c}
\frac{n}{2}-i \\
2
\end{array}\right)\right] \mid \leq 1 \\
& \quad \Rightarrow \frac{1}{2}|2 i(n-m)-(m-1)| \leq 1 \\
& \Rightarrow m-3 \leq 2 i(n-m) \leq m+1, \text { where } i=\left[\frac{m-1}{6}\right] .
\end{aligned}
$$

(4) Since $m+n$ is odd, $\left|V\left(K_{m} \cdot K_{n}\right)\right|$ is even. Therefore every friendly labeling satisfying the condition $\left|v_{f}(0)-v_{f}(1)\right|=0$. Consider the partition of $m$ and $n$ as $\left(\frac{m}{2}-i, \frac{m}{2}+i\right)$ and $\left(\frac{n+(2 i+1)}{2}-1, \frac{n-(2 i+1)}{2}+1\right)$ respectively, where $i=1,2,3, \ldots, \frac{m}{2}$.

Then $K_{m} \cdot K_{n}$ is balanced if

$$
\begin{aligned}
& \mid\left[\left(\begin{array}{c}
\frac{m}{2}-i \\
2
\end{array}\right)+\left(\frac{n+(2 i+1)}{2}-1\right)\right] \\
& \quad-\left[\left(\begin{array}{c}
\frac{m}{2}+i \\
2
\end{array}\right)+\left(\frac{n-(2 i+1)}{2}+1\right)\right] \mid \leq 1,
\end{aligned}
$$

$\Rightarrow \frac{1}{2}|2 i(n-m)-(n-1)| \leq 1$,

$\Rightarrow n-3 \leq 2 i(n-m) \leq n+1, i=1,2,3, \ldots, \frac{m}{2}$.

\section{BALANCE INDEX SET OF ONE POINT UNION OF TWO COMPLETE GRAPHS}

Now we are discussing the Balance index set of $K_{m} \cdot K_{n}$

THEOREM 5. The balance index set of $K_{m} \cdot K_{n}$ (where $m \leq n)$ is

(1)

$$
\begin{aligned}
& \left\{\mid \frac{(2 i-1) n-(2 i+1) m}{2}\right. \\
& \left.\quad+1 \mid, \frac{(2 i+1)|n-m|}{2}: i=0,1,2, \ldots, \frac{m-1}{2}\right\} \bigcup \\
& \left\{\left|\frac{(2 i+3) n-(2 i+1) m}{2}-1\right|: i=0,1,2, \ldots, \frac{m-3}{2}\right\},
\end{aligned}
$$

(2) $\left\{|i(n-m)-(n-1)|, i|(n-m)|: i=0,1,2, \ldots \frac{m}{2}\right\} \bigcup$ $\left\{\mid i(n-m)+(n-1): i=0,1,2, \ldots \frac{m}{2}-1\right\}$, if $m$ and $n$ are even.

(3) $\left\{\frac{|2 i(n-m)-(m-1)|}{2}: i=0,1,2, \ldots \frac{m-1}{2}\right\} \bigcup$ $\left\{\frac{|2(i+1)(n-m)+(m-1)|}{2}: i=0,1,2, \ldots \frac{m-3}{2}\right\}$,
if $m+n=$ odd and $\min \{m, n\}$ is odd.

(4) $\left\{\frac{|2 i(n-m)-(n-1)|}{2}: i=0,1,2, \ldots \frac{m}{2}\right\} \bigcup$ $\left\{\frac{|2 i(n-m)+(n-1)|}{2}: i=0,1,2, \ldots \frac{m}{2}-1\right\}$, if $m+n=$ odd and $\min \{m, n\}$ is even.

PROOF. (1) If $m$ and $n$ are odd.

The possible distinct partitions of $m$ and $n$, for friendly labeling given in table 1 .

Case 1. If the partition of $m$ and $n$ are

$$
\begin{aligned}
& \left(\frac{m-(2 i+1)}{2}, \frac{m+(2 i+1)}{2}\right) \text { and } \\
& \left(\frac{n+(2 i+1)}{2}-1, \frac{n-(2 i+1)}{2}+1\right),
\end{aligned}
$$

where $i=0,1,2, \ldots, \frac{m-1}{2}$.

Then $\left|e_{f^{*}}(0)-e_{f^{*}}(1)\right|=$

$$
\begin{aligned}
& \mid\left[\left(\frac{m-(2 i+1)}{2}\right)+\left(\frac{n+(2 i+1)}{2}-1\right)\right] \\
& \quad-\left[\left(\frac{m+(2 i+1)}{2}\right)+\left(\frac{n-(2 i+1)}{2}+1\right)\right] \mid
\end{aligned}
$$

$=\left|\frac{(2 i-1) n-(2 i+1) m}{2}+1\right|$,

where $i=0,1,2, \ldots, \frac{m-1}{2}$.

Case 2. If the partition of $m$ and $n$ are

$\left(\frac{m-(2 i+1)}{2}, \frac{m+(2 i+1)}{2}\right)$ and

$\left(\frac{n+(2 i+1)}{2}, \frac{n-(2 i+1)}{2}\right)$, where $i=0, \quad 1, \quad 2$,

$\ldots, \frac{m-1}{2}$.

Then $\left|e_{f^{*}}(0)-e_{f^{*}}(1)\right|=$

$$
\begin{gathered}
\mid\left[\left(\frac{m-(2 i+1)}{2}\right)+\left(\frac{n+(2 i+1)}{2}\right)\right] \\
-\left[\left(\frac{m+(2 i+1)}{2}\right)+\left(\frac{n-(2 i+1)}{2}\right)\right] \mid \\
=\frac{(2 i+1)|n-m|}{2}, \text { where } i=0,1,2, \ldots, \frac{m-1}{2} .
\end{gathered}
$$

Case 3. If the partition of $m$ and $n$ are

$\left(\frac{m-(2 i+1)}{2}, \frac{m+(2 i+1)}{2}\right)$ and

$\left(\frac{n+(2 i+1)}{2}+1, \frac{n-(2 i+1)}{2}-1\right)$,

where $i=0,1,2, \ldots, \frac{m-3}{2}$. 
Table 1. Possible distinct partitions of $\mathbf{m}$ and $\mathbf{n}$, for friendly labeling.

\begin{tabular}{|c|c|}
\hline$m$ & $n$ \\
\hline \multirow{2}{*}{$\left(\frac{m-(2 i+1)}{2}, \frac{m+(2 i+1)}{2}\right)$} & $\left(\frac{n+(2 i+1)}{2}-1, \frac{n-(2 i+1)}{2}+1\right)$ and $\left(\frac{n+(2 i+1)}{2}, \frac{n-(2 i+1)}{2}\right), i=0,1,2, \ldots, \frac{m-1}{2}$ \\
\cline { 2 - 2 } & $\left(\frac{n+(2 i+1)}{2}+1, \frac{n-(2 i+1)}{2}-1\right), i=0,1,2, \ldots, \frac{m-3}{2}$ \\
\hline
\end{tabular}

Then $\left|e_{f^{*}}(0)-e_{f^{*}}(1)\right|=$

$$
\begin{aligned}
& \qquad \mid\left[\left(\frac{m-(2 i+1)}{2}\right)+\left(\frac{n+(2 i+1)}{2}+1\right)\right] \\
& \qquad-\left[\left(\frac{m+(2 i+1)}{2}\right)+\left(\frac{n-(2 i+1)}{2}-1\right)\right] \mid \\
& =\left|\frac{(2 i+3) n-(2 i+1) m}{2}-1\right|, \\
& \text { where } i=0,1,2, \ldots, \frac{m-3}{2} . \\
& \text { Therefore } \mathrm{BI}\left(K_{m} \cdot K_{n}\right)= \\
& \qquad \mid \frac{(2 i-1) n-(2 i+1) m}{2} \\
& \left.\quad+1 \mid, \frac{(2 i+1)|n-m|}{2}: i=0,1,2, \ldots, \frac{m-1}{2}\right\} \\
& \cup\left\{\left|\frac{(2 i+3) n-(2 i+1) m}{2}-1\right|: i=0,1,2, \ldots, \frac{m-3}{2}\right\} .
\end{aligned}
$$

(2) If $m$ and $n$ are even.

The possible distinct partitions of $m$ and $n$, for friendly labeling given in table 2 .

Case 1. If the partition of $m$ and $n$ are

$\left(\frac{m}{2}-i, \frac{m}{2}+i\right)$ and $\left(\frac{n}{2}+i-1, \frac{n}{2}-i+1\right)$, where $i=0,1,2, \ldots, \frac{m}{2}$.

Then $\left|e_{f^{*}}(0)-e_{f^{*}}(1)\right|=$

$$
\begin{gathered}
\mid\left[\left(\begin{array}{c}
\frac{m}{2}-i \\
2
\end{array}\right)+\left(\begin{array}{c}
\frac{n}{2}+i-1 \\
2
\end{array}\right)\right] \\
-\left[\left(\begin{array}{c}
\frac{m}{2}+i \\
2
\end{array}\right)+\left(\begin{array}{c}
\frac{n}{2}-i+1 \\
2
\end{array}\right)\right] \mid \\
=|i(n-m)-(n-1)|, \text { where } i=0,1,2, \ldots, \frac{m}{2} .
\end{gathered}
$$

Case 2. If the partition of $m$ and $n$ are $\left(\frac{m}{2}-i, \frac{m}{2}+i\right)$ and $\left(\frac{n}{2}+i, \frac{n}{2}-i\right)$, where $i=0,1,2, \ldots, \frac{m}{2}$.

Then $\left|e_{f^{*}}(0)-e_{f^{*}}(1)\right|=$

$\left|\left[\left(\begin{array}{c}\frac{m}{2}-i \\ 2\end{array}\right)+\left(\begin{array}{c}\frac{n}{2}+i \\ 2\end{array}\right)\right]-\left[\left(\begin{array}{c}\frac{m}{2}+i \\ 2\end{array}\right)+\left(\begin{array}{c}\frac{n}{2}-i \\ 2\end{array}\right)\right]\right|$

$=i|n-m|$, where $i=0,1,2, \ldots, \frac{m}{2}$.

Case 3. If the partition of $m$ and $n$ are

$\left(\frac{m}{2}-i, \frac{m}{2}+i\right)$ and $\left(\frac{n}{2}+i+1, \frac{n}{2}-i-1\right)$, where $i=0,1,2, \ldots, \frac{m}{2}-1$.

Then $\left|e_{f^{*}}(0)-e_{f^{*}}(1)\right|=$

$$
\begin{aligned}
& \mid\left[\left(\begin{array}{c}
\frac{m}{2}-i \\
2
\end{array}\right)+\left(\begin{array}{c}
\frac{n}{2}+i+1 \\
2
\end{array}\right)\right] \\
& \quad-\left[\left(\begin{array}{c}
\frac{m}{2}+i \\
2
\end{array}\right)+\left(\begin{array}{c}
\frac{n}{2}-i-1 \\
2
\end{array}\right)\right] \mid
\end{aligned}
$$

$=|i(n-m)+(n-1)|$, where $i=0,1,2, \ldots, \frac{m}{2}-1$.

Therefore $\operatorname{BI}\left(K_{m} \cdot K_{n}\right)=$

$\left\{|i(n-m)-(n-1)|, i|(n-m)|: i=0,1,2, \ldots, \frac{m}{2}\right\}$

$\bigcup\left\{\mid i(n-m)+(n-1): i=0,1,2, \ldots, \frac{m}{2}-1\right\}$

(3) If $m$ is odd and $n$ is even.

The possible distinct partitions of $m$ and $n$, for friendly labeling given in table 3 .

Case 1. If the partition of $m$ and $n$ are $\left(\frac{m-(2 i+1)}{2}, \frac{m+(2 i+1)}{2}\right)$ and $\left(\frac{n}{2}+i, \frac{n}{2}-i\right)$, where $i=0,1,2, \ldots, \frac{m-1}{2}$.

Then $\left|e_{f^{*}}(0)-e_{f^{*}}(1)\right|=$

$$
\begin{aligned}
& \mid\left[\left(\begin{array}{c}
\frac{m-(2 i+1)}{2} \\
2
\end{array}\right)+\left(\begin{array}{c}
\frac{n}{2}+i \\
2
\end{array}\right)\right] \\
& -\left[\left(\begin{array}{c}
\frac{m+(2 i+1)}{2} \\
2
\end{array}\right)+\left(\begin{array}{c}
\frac{n}{2}-i \\
2
\end{array}\right)\right] \mid
\end{aligned}
$$

$=\left|\frac{2 i(n-m)-(m-1)}{2}\right|$, where $i=0,1,2, \ldots, \frac{m-1}{2}$.

Case 2. If the partition of $m$ and $n$ are

$\left(\frac{m-(2 i+1)}{2}, \frac{m+(2 i+1)}{2}\right)$ and

$\left(\frac{n}{2}+i+1, \frac{n}{2}-i-1\right)$, where $i=0,1,2, \ldots, \frac{m-3}{2}$.

Then $\left|e_{f^{*}}(0)-e_{f^{*}}(1)\right|=$

$$
\begin{aligned}
& \mid\left[\left(\begin{array}{c}
\frac{m-(2 i+1)}{2} \\
2
\end{array}\right)+\left(\begin{array}{c}
\frac{n}{2}+i+1 \\
2
\end{array}\right)\right] \\
& -\left[\left(\begin{array}{c}
\frac{m+(2 i+1)}{2} \\
2
\end{array}\right)+\left(\begin{array}{c}
\frac{n}{2}-i-1 \\
2
\end{array}\right)\right] \mid
\end{aligned}
$$

$=\left|\frac{2(i+1)(n-m)+(m-1)}{2}\right|$,

where $i=0,1,2, \ldots, \frac{m-3}{2}$.

Therefore $\operatorname{BI}\left(K_{m} \cdot K_{n}\right)=$

$\left\{\left|\frac{2 i(n-m)-(m-1)}{2}\right|: i=0,1,2, \ldots, \frac{m-1}{2}\right\} \bigcup$
$\left\{\left|\frac{(2(i+1)(n-m)+(m-1)}{2}\right|: i=0,1,2, \ldots, \frac{m-3}{2}\right\}$.

(4) If $m$ is even and $n$ is odd.

The possible distinct partitions of $m$ and $n$, for friendly labeling given in table 4.

Case 1. If the partition of $m$ and $n$ are $\left(\frac{m}{2}-i, \frac{m}{2}+i\right)$ and $\left(\frac{n+(2 i+1)}{2}-1, \frac{n-(2 i+1)}{2}+1\right)$, where $i=0,1,2, \ldots, \frac{m}{2}$. 
Table 2. Possible distinct partitions of $m$ and $\mathbf{n}$, for friendly labeling.

\begin{tabular}{|c|c|}
\hline$m$ & $n$ \\
\hline \multirow{2}{*}{$\left(\frac{m}{2}-i, \frac{m}{2}+i\right)$} & $\left(\frac{n}{2}+i-1, \frac{n}{2}-i+1\right)$ and $\left(\frac{n}{2}+i, \frac{n}{2}-i\right), i=0,1,2, \ldots, \frac{m}{2}$ \\
\cline { 2 - 2 } & $\left(\frac{n}{2}+i+1, \frac{n}{2}-i-1\right), i=0,1,2, \ldots, \frac{m}{2}-1$ \\
\hline
\end{tabular}

Table 3. Possible distinct partitions of $m$ and $n$, for friendly labeling.

\begin{tabular}{|c|c|}
\hline$m$ & $n$ \\
\hline \multirow{2}{*}{$\left(\frac{m-(2 i+1)}{2}, \frac{m+(2 i+1)}{2}\right)$} & $\left(\frac{n}{2}+i, \frac{n}{2}-i\right), i=0,1,2, \ldots, \frac{m-1}{2}$ \\
\cline { 2 - 2 } & $\left(\frac{n}{2}+i+1, \frac{n}{2}-i-1\right), i=0,1,2, \ldots, \frac{m-3}{2}$ \\
\hline
\end{tabular}

Table 4. Possible distinct partitions of $m$ and $n$, for friendly labeling.

\begin{tabular}{|c|c|}
\hline$m$ & $n$ \\
\hline \multirow{3}{*}{$\left(\frac{m}{2}-i, \frac{m}{2}+i\right)$} & $\left(\frac{n+(2 i+1)}{2}-1, \frac{n-(2 i+1)}{2}+1\right), i=0,1,2, \ldots, \frac{m}{2}$ \\
\cline { 2 - 2 } & $\left(\frac{n+(2 i+1)}{2}, \frac{n-(2 i+1)}{2}\right), i=0,1,2, \ldots, \frac{m}{2}-1$ \\
\hline
\end{tabular}

Then $\left|e_{f^{*}}(0)-e_{f^{*}}(1)\right|=$

$$
\begin{aligned}
\mid & {\left[\left(\begin{array}{c}
\frac{m}{2}-i \\
2
\end{array}\right)+\left(\frac{n+(2 i+1)}{2}-1\right)\right] } \\
- & {\left[\left(\begin{array}{c}
\frac{m}{2}+i \\
2
\end{array}\right)+\left(\frac{n-(2 i+1)}{2}+1\right)\right] \mid } \\
= & \left|\frac{2 i(n-m)-(n-1)}{2}\right|, \text { where } i=0,1,2, \ldots, \frac{m}{2} .
\end{aligned}
$$

Case 2. If the partition of $m$ and $n$ are $\left(\frac{m}{2}-i, \frac{m}{2}+i\right)$ and $\left(\frac{n+(2 i+1)}{2}, \frac{n-(2 i+1)}{2}\right)$, where $i=0,1,2$, ..., $\frac{m}{2}-1$.

Then $\left|e_{f^{*}}(0)-e_{f^{*}}(1)\right|=$

$$
\begin{aligned}
& \qquad \mid\left[\left(\begin{array}{c}
\frac{m}{2}-i \\
2
\end{array}\right)+\left(\frac{n+(2 i+1)}{2}\right)\right] \\
& -\left[\left(\begin{array}{c}
\frac{m}{2}+i \\
2
\end{array}\right)+\left(\begin{array}{c}
\frac{n-(2 i+1)}{2} \\
2
\end{array}\right)\right] \mid \\
& =\left|\frac{2 i(n-m)+(n-1)}{2}\right|, \text { where } i=0,1,2, \ldots, \frac{m}{2}-1 . \\
& \text { Therefore } \mathrm{BI}\left(K_{m} \cdot K_{n}\right)=
\end{aligned}
$$

$$
\begin{aligned}
& \left\{\left|\frac{2 i(n-m)-(n-1)}{2}\right|: i=0,1,2, \ldots, \frac{m}{2}\right\} \bigcup \\
& \left\{\left|\frac{(2 i(n-m)+(n-1)}{2}\right|: i=0,1,2, \ldots, \frac{m}{2}-1\right\} .
\end{aligned}
$$

\section{CONCLUSION}

Labeled graph is the topic of current interest for many researchers as it has diversified applications. We discuss here balanced labeling and balance index set of one point union of two complete graphs. The derived labeling pattern is demonstrated by means of elegant illustrations which provides better understanding of the derived results. This study can be extended to chain graphs whose blocks are complete and its compliment.

\section{REFERENCES}

[1] L. W. Beineke and S. M. Hegde. Strongly multiplicative graphs. Discuss. Math. Graph Theory, 21:63-75, 2001.

[2] I. Cahit. Cordial graphs: a weaker version of graceful and harmonious graphs. Ars Combin., 23:201-207, 1987.

[3] J. A. Gallian. A dynamic survey of graph labeling. The Electronics Journal of Combinatorics, 16(DS6), 2009.

[4] Frank Harary. Graph Theory. Narosa Publishing House, 1989.

[5] R. Y. Kim, S-M. Lee, and H. K. Ng. On balancedness of some family of graphs. Manuscript.

[6] Alexander Nien-Tsu Lee, Sin-Min Lee, and Ho Kuen Ng. On the balance index set of graphs. J.Combin. Math. Combin. Comp., 66:135-150, 2008.

[7] S-M. Lee, A. Liu, and S.K. Tan. On balanced graphs. J.Combin. Math. Combin. Comp., 87:59-64, 2008. 